\section{Metastatic Anaplastic Lymphoma Kinase Rearrangement-Positive Adenocarcinoma of Occult Primary Mimicking Ovarian Cancer}

\author{
Michael Chahin ${ }^{1}$, Nithya Krishnan ${ }^{1}$, Trevanne Matthews-Hew ${ }^{2}$, Jason Hew $^{3}$, Dat Pham ${ }^{2}$ \\ 1. Internal Medicine, University of Florida College of Medicine - Jacksonville, Jacksonville, USA 2. Hematology and \\ Oncology, University of Florida College of Medicine - Jacksonville, Jacksonville, USA 3. Hematology and Oncology, \\ 21st Century Oncology, Jacksonville, USA
}

Corresponding author: Michael Chahin, michael.chahin@jax.ufl.edu

\begin{abstract}
Lung cancer is a worldwide concern and is the leading cause of cancer-related death in the United States. Adenocarcinoma is the most common type of non-small cell lung cancer; however, unlike other types of lung cancer this disease is often seen in light tobacco smokers and non-smokers. The presence of driver mutations, such as the epidermal growth factor receptor (EGFR) and echinoderm microtubule-associated protein-like 4 (EML-4)-anaplastic lymphoma kinase (ALK) rearrangement, appears to be more common in these patients. The presence of the ALK mutation provides a target for ALK-inhibiting agents, such as alectinib. Routine testing for driver mutations is the standard of care in the management of advanced nonsmall cell lung cancer.
\end{abstract}

Lung cancer frequently metastasizes to distant sites such as the bone, brain, and the adrenal glands, but rarely to the ovaries. We present a young, female, patient who complained of shortness of breath and was found to have pulmonary emboli, extensive lymphadenopathy, and a right ovarian mass. Initial pathology from a cervical lymph node favored a gastrointestinal or an ovarian malignancy. However, immunohistochemical staining ultimately suggested an occult lung adenocarcinoma primary with ovarian metastasis. She had a left oophorectomy that demonstrated similar findings and was positive for the ALK mutation. She was treated with alectinib with good response though ultimately died from her disease.

This case demonstrates the rare finding of an ALK-mutated lung adenocarcinoma with ovarian metastasis and, to our knowledge, it is the first with an occult lung adenocarcinoma primary. Driver mutation testing should be considered in metastasis from an occult primary when a pulmonary malignancy is suspected.

Categories: Pathology, Oncology, Pulmonology

Keywords: alk inhibitor, alectinib, lung, adenocarcinoma, ovary, metastasis

\section{Introduction}

Lung cancer is the most diagnosed cancer worldwide, and the primary risk factor is tobacco consumption. Some other risk factors include exposure to certain toxins, increased age, genetic factors, and infections such as HIV $[1,2]$. Historically, squamous cell carcinoma was the most frequent subtype of non-small cell lung cancer (NSCLC), but newer studies show adenocarcinoma as the most common [3]. Many patients who develop adenocarcinoma of the lung have no smoking history. These patients are frequently younger and present with advanced disease. Epidermal growth factor receptor (EGFR) and echinoderm microtubuleassociated protein-like 4 (EML-4)-anaplastic lymphoma kinase (ALK) mutations are often found in individuals with adenocarcinoma and no, or light $(\leqslant 10$ pack-years), smoking history $[2,4]$.

An autopsy study revealed the three most common sites of hematogenous metastasis of lung cancer to be the liver, adrenal glands, and bone. Out of the 175 individuals who had lung cancer metastasis, $<1 \%$ had ovarian involvement [5]. A case series demonstrated that lung adenocarcinomas with ovarian metastasis may frequently harbor the EML4-ALK mutation, thus providing a target for therapy in patients with advanced disease [6].

\section{Case Presentation}

A 29-year African American female with no smoking history presented with right lower extremity pain and swelling, and shortness of breath. She had a four-day history of productive cough without hemoptysis and a 45 pound weight loss over the past few months. She denied oral contraceptive use or tobacco use. Her physical exam was remarkable for $1+$ pitting edema and tenderness to palpation of the right calf and thigh. She had right cervical adenopathy and a muffled voice. She was tachycardic but normotensive. Eastern Cooperative Oncology Group performance status was 1. 


\section{Cureus}

CT of the soft tissue neck with contrast revealed enlarged necrotic cervical, bilateral supraclavicular, and superior mediastinal lymph nodes. CT angiography showed pulmonary emboli and right peribronchial thickening without a definitive mass. A subsequent $\mathrm{CT}$ of the abdomen and pelvis revealed right common femoral deep venous thrombosis and a right adnexal mass (Figure 1).

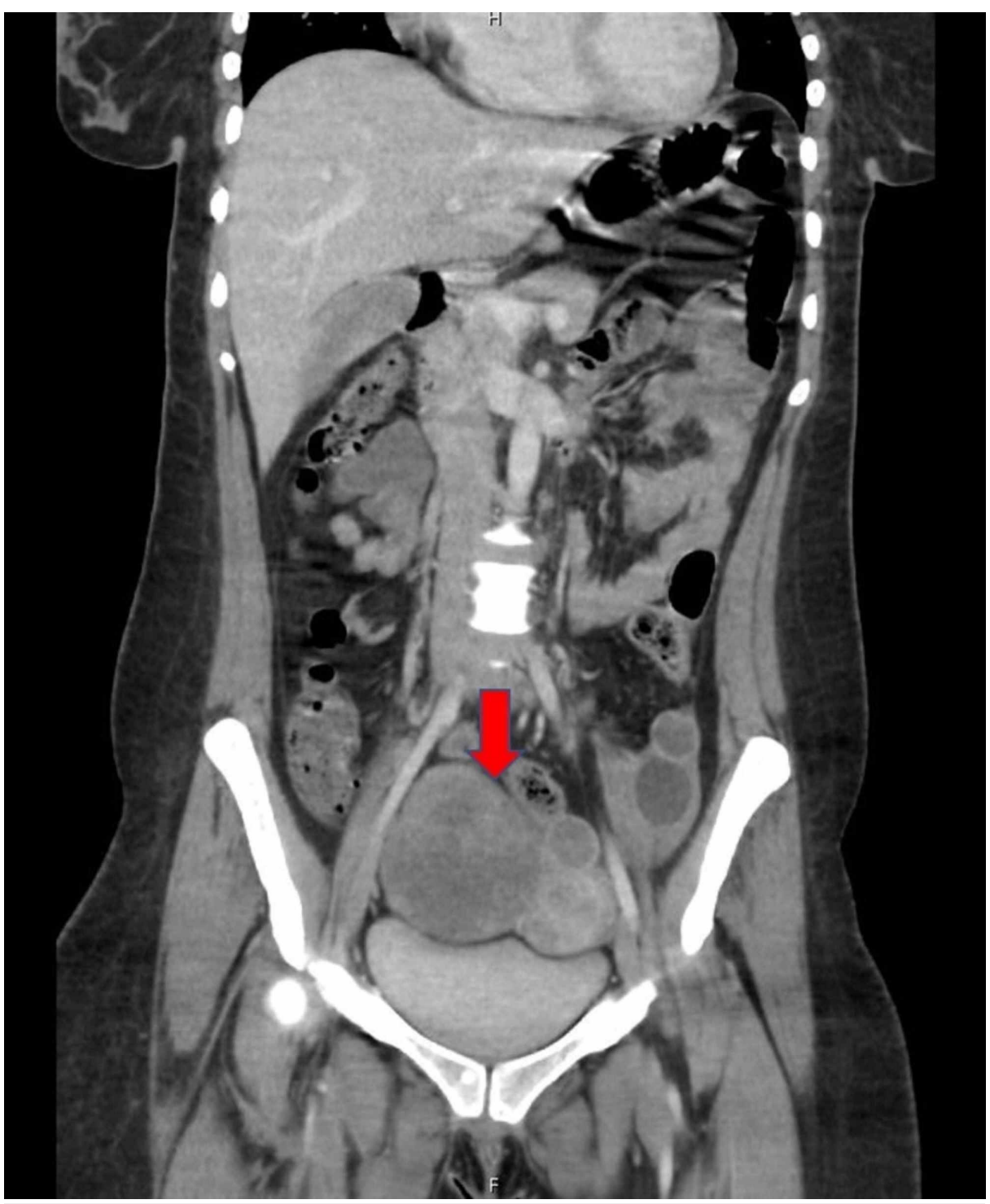

FIGURE 1: Coronal view of the abdomen and pelvis CT demonstrating a $9.5 \mathrm{~cm}$ solid and cystic right adnexal mass.

Biopsy of the right cervical lymph node revealed metastatic, poorly differentiated adenocarcinoma. Immunohistochemical staining showed cytokeratin (CK) AE1/AE3, CK7, thyroid transcription factor (TTF) 1 , and Napsin A positivity, consistent with a lung primary. Negative immunostains included CK20, CK 5/6, thyroglobulin, calcitonin, CD56, PLAP, chromogranin, cancer antigen (CA) 19.9, CD45, and S100. She underwent left adnexal oophorectomy, from which pathology later revealed $100 \%$ ALK positive poorly differentiated adenocarcinoma of pulmonary origin, programmed cell death protein 1 (PD-1) 30\% on immunohistochemical assay (Figures 2, 3). 


\section{Cureus}

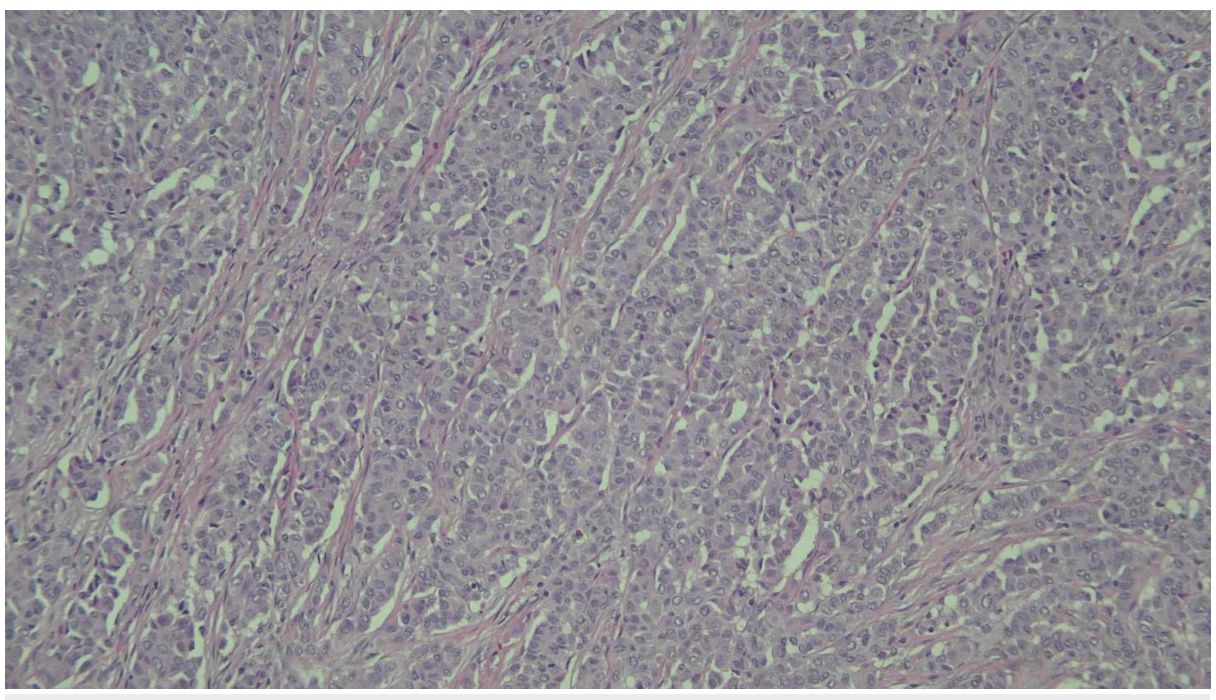

FIGURE 2: Poorly differentiated adenocarcinoma involving the ovary (H\&E stain, $\times 100$ ).

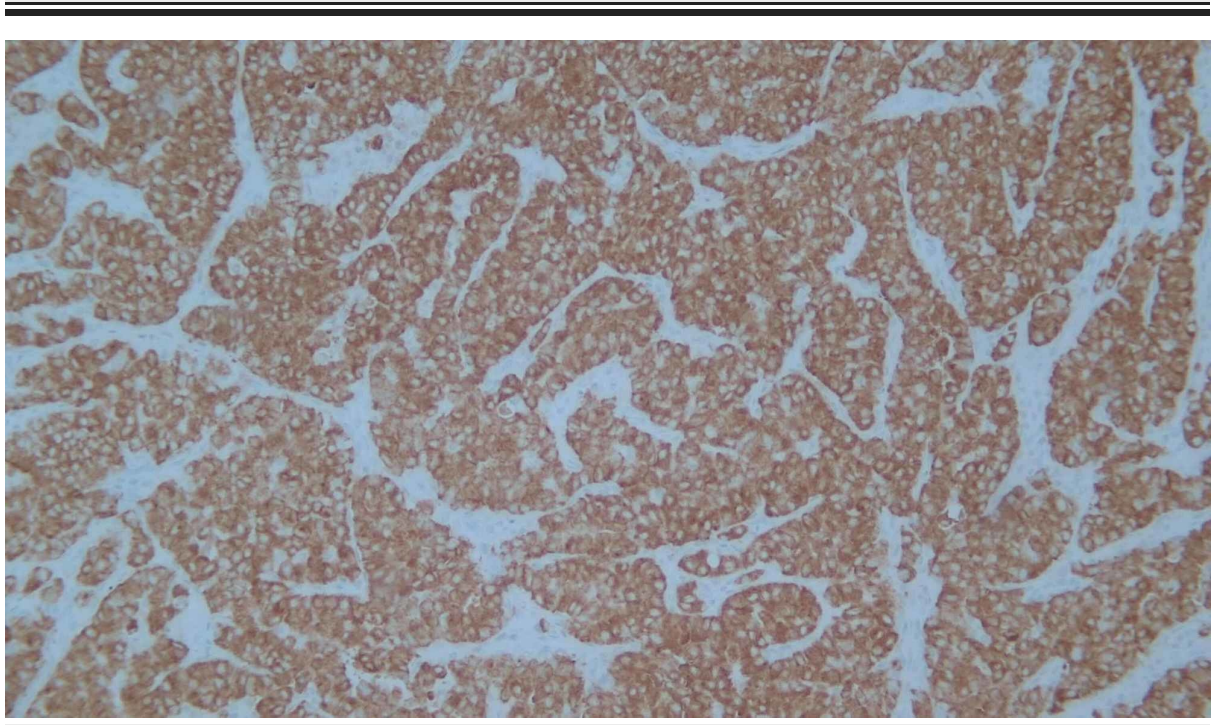

FIGURE 3: Poorly differentiated adenocarcinoma involving the ovary. The tumor cells are immunoreactive for ALK (ALK (D5F3) VENTANA FDA CDx assay, $\times 100$ ).

ALK, anaplastic lymphoma kinase

A staging brain MRI revealed a metastatic parietal lobe lesion. After receiving stereotactic radiotherapy for brain metastasis, the patient was started on alectinib $600 \mathrm{mg}$ twice daily with minimal adverse effects.

Her initial CA 125 was 3,641 U/mL and carcinoembryonic antigen (CEA) was $52.1 \mathrm{ng} / \mathrm{mL}$. After six months of therapy, her markers had decreased to $129 \mathrm{U} / \mathrm{mL}$ and $10.7 \mathrm{ng} / \mathrm{mL}$, respectively. An initial position emission tomography (PET)/CT was not performed, but a follow-up study CT around this time revealed a slight decrease of the right ovarian mass to $7.1 \mathrm{~cm}$ and diffuse bony lesions (Figure 4). It was unclear if the bone lesions were new given the lack of prior imaging. 


\section{Cureus}

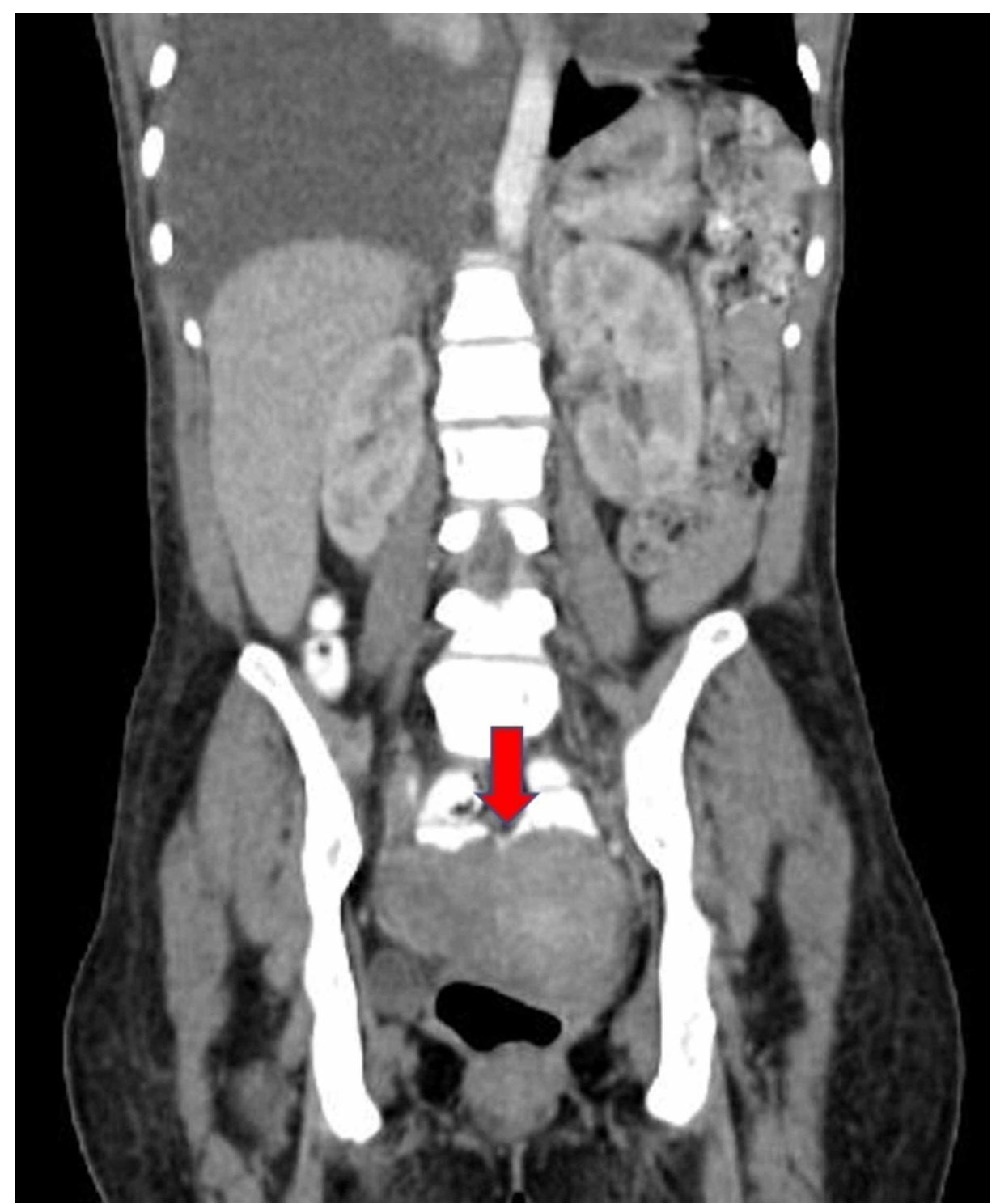

FIGURE 4: Coronal view of the abdomen and pelvis CT demonstrating a heterogeneous partially cystic right adnexal lesion, six months after starting alectinib.

After eight months of alectinib therapy, her CA 125 and CEA levels increased. She had increased pain from bony metastasis and required repeated therapeutic thoracentesis of a right-side malignant pleural effusion. Cytology was positive for metastatic adenocarcinoma. She was then switched to pembrolizumab, a PD-1 inhibitor, $200 \mathrm{mg}$ every 21 days due to symptomatic progression of disease with multiple, systemic, lesions. Had she had been asymptomatic, had symptomatic brain recurrence only, or had an isolated lesion she would have been a candidate for continuation of alectinib.

She went on to have pathologic fracture of a thoracic vertebrae and received radiotherapy for her osseous metastases. By the time of follow-up visit three months from starting pembrolizumab, she was wheelchairbound and requiring supplemental oxygen. The plan was to switch to brigatinib, also an ALK inhibitor, due to continued disease progression. At the time it had been approved for patients who progressed on crizotinib. Unfortunately, however, she died approximately two weeks later, prior to starting therapy.

\section{Discussion}

On initial pathology review from the cervical lymph node biopsy, a primary ovarian malignancy and upper GI primary were strongly considered. No definitive lung mass was seen but the immunohistochemistry staining pattern was consistent with a lung primary, based on CK7, TTF1, and Napsin A positivity. 
to chemotherapy for ALK-positive NSCLC [7]. Alectinib, a highly selective ALK inhibitor, was approved initially in 2015 for patients who had progressed on or did not tolerate crizotinib and became first line in 2017, following the alectinib versus crizotinib in untreated ALK-positive non-small cell lung cancer (ALEX) trial. Patients treated with alectinib had a response rate of $82.9 \%$, compared to $75.5 \%$ in those treated with crizotinib [8,9]. The alectinib versus chemotherapy in crizotinib-pretreated ALK-positive non-small cell lung cancer (ALUR) trial showed higher progression-free survival in patients treated with alectinib compared to chemotherapy, 9.6 months vs 1.6 months, in patients who had progressed after platinum-based chemotherapy and crizotinib [10].

For patients who progress on an ALK inhibitor and have multiple symptomatic lesions, lorlatinib, also an ALK inhibitor, may be used as can PD-1 targeting agents such as pembrolizumab [11]. Pembrolizumab has shown promise in overall survival in NSCLC patients whose tumors express PD ligand (L)1 (>50\%) [12]. At the time of this patient's diagnosis, alectinib had only recently been approved as first-line therapy and lorlatinib was not approved in the USA at the time [13].

ALK rearrangement is found in approximately $7 \%$ of lung adenocarcinomas, compared to the EGFR mutation which is seen in approximately $15 \%[14,15]$. The case series and literature review by Bi et al. reveals a possible association between ALK rearrangement positivity and ovarian metastasis from lung adenocarcinoma primary. This series accounts for, to our knowledge, all published cases of patients who developed ovarian metastasis from lung adenocarcinoma with ALK mutation. Out of the 16 cases evaluated in these case series, 11 of the 16 patients, or $69 \%$, had the ALK rearrangement. Two of the 16 patients had EGFR mutation, suggesting the ALK mutation is the most common mutation in these lung cancer patients with ovarian metastasis [6]. The present case is unique from the other published reports due to this patient developing ovarian metastasis from an occult lung primary.

Patients presenting with advanced disease should have molecular testing for mutations such as ALK and PD1 [11]. Despite having extensive disease at diagnosis, this patient had an initial positive response with alectinib. Her course was complicated by multiple hospitalizations due to shortness of breath and failure to thrive, causing delay in care. An initial PET/CT was never done, making imaging somewhat unreliable for monitoring progression. The solid portion of the ovarian mass decreased in size, but bone metastases developed. The primary objective findings in this case of good response are the tumor markers. At the time of progression of her disease her functional status had deteriorated making her not a candidate for chemotherapy.

\section{Conclusions}

Ovarian metastasis from lung adenocarcinoma is a rare entity and seems to be associated with EML4-ALK rearrangement mutation. This case reinforces that molecular testing should be performed on all patients with systemic NSCLC. This patient presented with advanced, metastatic disease but had a good response to ALK-inhibiting therapy.

\section{Additional Information \\ Disclosures}

Human subjects: Consent was obtained by all participants in this study. Conflicts of interest: In compliance with the ICMJE uniform disclosure form, all authors declare the following: Payment/services info: All authors have declared that no financial support was received from any organization for the submitted work. Financial relationships: All authors have declared that they have no financial relationships at present or within the previous three years with any organizations that might have an interest in the submitted work. Other relationships: All authors have declared that there are no other relationships or activities that could appear to have influenced the submitted work.

\section{References}

1. Bray F, Ferlay J, Soerjomatarm I, Siegal RL, Torre LA, Jemal A: Global cancer statistics 2018: GLOBOCAN estimates of incidence and mortality worldwide for 36 cancers in 185 countries. CA Cancer J Clin. 2018, 68:394-424. 10.3322/caac.21492

2. De Groot PM, Wu CC, Carter BW, Munden RF: The epidemiology of lung cancer. Transl Lung Cancer Res. 2018, 7:220-233. 10.21037/tlcr.2018.05.06

3. Tazelaar HD: Pathology of Lung Malignancies. Vora SR (ed): UpToDate, Waltham, MA; 2019.

4. Shaw A, Yeap B, Mino-Kenudson M, et al.: Clinical features and outcome of patients with non-small-cell lung cancer who harbor EML4-ALK. J Clin Oncol. 2009, 27:4247-4253. 10.1200/JCO.2009.22.6993

5. Milovanovic IS, Stjepanovic M, Mitrovic D: Distribution patterns of the metastases of the lung carcinoma in relation to histological type of the primary tumor: an autopsy study. Ann Thorac Med. 2017, 12:191-198. 10.4103/atm.ATM_276_16

6. Bi R, Bai Q, Zhu X, et al.: ALK rearrangement: a high-frequency alteration in ovarian metastasis from lung adenocarcinoma. Diagn Pathol. 2019, 14:96. 10.1186/s13000-019-0864-7

7. Khan M, Lin J, Liao G, et al.: ALK inhibitors in the treatment of ALK positive NSCLC . Front Oncol. 2018, 8:557. 10.3389/fonc.2018.00557

8. Barrows SM, Wright K, Copley-Merriman C, et al.: Systematic review of sequencing of ALK inhibitors in 


\section{Cureus}

ALK-positive non-small-cell lung cancer. Lung Cancer. 2019, 10:11-20. 10.2147/LCTT.S179349

9. Peters S, Cambidge DR, Shaw AT, et al.: Alectinib versus crizonitib in untreated ALK-positive non-small cell lung cancer. N Engl J Med. 2017, 377:829-838. 10.1056/NEJMoa1704795

10. Novello S, Mazieres J, Oh IJ, et al.: Alectinib versus chemotherapy in crizotinib-pretreated anaplastic lymphoma kinase (ALK)-positive non-small-cell lung cancer: results from the phase III ALUR study. Ann Oncol. 2018, 29:1409-1416. 10.1093/annonc/mdy121

11. National Comprehensive Cancer Network. Non-small cell lung cancer (version 3) . (2020). Accessed: June 15, 2020: https://www.nccn.org/professionals/physician_gls/default.aspx.

12. Lim SH, Sun JM, Lee SH, Ahn JS, Park K, Ahn MJ: Pembrolizumab for the treatment of non-small cell lung cancer. Expert Opin Biol Ther. 2016, 16:397-406. 10.1517/14712598.2016.1145652

13. Syed YY: Loratinib: first global approval . Drugs. 2019, 79:93-98. 10.1007/s40265-018-1041-0

14. Soda M, Choi YL, Enomoto M, et al.: Identification of the transforming EML4-ALK fusion gene in non-smallcell lung cancer. Nature. 2007, 448:561-566. 10.1038/nature05945

15. Barlesi F, Mazieres J, Merlio JP, et al.: Routine molecular profiling of patients with advanced non-small-cell lung cancer: results of a 1-year nationwide programme of the French Cooperative Thoracic Intergroup (IFCT). Lancet. 2016, 387:1425-1426. 10.1016/S0140-6736(16)00004-0 